\title{
Biodiversidad e hipovirulencia de Cryphonectria parasitica en Europa: implicaciones para el control biológico del cancro del castaño
}

Recibido: 6 xullo 2011 / Aceptado: 20 setembro 2011

(C) IBADER- Universidade de Santiago de Compostela 2011

\begin{abstract}
Resumen El cancro del castaño, causado por Cryphonectria parasitica, es una enfermedad ampliamente extendida en todo el mundo. En Europa, se ha detectado en la mayoría de las zonas cultivadas con Castanea sativa (castaño europeo) en países mediterráneos y centroeuropeos, y se considera un patógeno de cuarentena. No existe ningún método cultural ni químico de control de este hongo, ni ningún cultivar de castaño europeo tolerante o resistente a la enfermedad. En los últimos años, la investigación sobre el control del cancro se ha centrado en el desarrollo de métodos biológicos. Cryphonectria parasitica presenta dos tipos de cepas: virulentas, que causan graves lesiones en el árbol, e hipovirulentas, que apenas ocasionan daños porque son portadoras de un virus que atenúa su virulencia. La aplicación en campo, sobre castaños afectados, de cepas hipovirulentas, que pueden transmitir su virus a las virulentas, es, por el momento, la única perspectiva para reducir y/o minimizar los daños que este patógeno ocasiona. El éxito de esta técnica de control biológico del cancro requiere un conocimiento previo de la estructura poblacional de Cryphonectria parasitica (número y distribución de los tipos de compatibilidad vegetativa y sexual) y la existencia de cepas hipovirulentas compatibles con las virulentas dominantes en una zona afectada.
\end{abstract}

Palabras clave Castanea sativa, virus ARNbc, compatibilidad vegetativa, tipos de apareamiento

Olga Aguín · Dolores Montenegro · J. Pedro Mansilla

Estación Fitopatolóxica do Areeiro, Deputación de Pontevedra,

Subida a la Robleda s/n, 36153 Pontevedra

Tel: 986 841491; Fax : 986864291

e-mail: olga.aguin@depo.es

María Jesús Sainz

Departamento de Producción Vegetal, Universidad de Santiago de Compostela, Campus Universitario s/n, 27002 Lugo
Abstract Chestnut blight, caused by Cryphonectria parasitica, is a widespread disease throughout the world. In Europe, it has been detected in most cultivated areas of Castanea sativa (European chestnut) in Mediterranean and Central European countries, and is considered a quarantine pathogen. There is no cultural or chemical method to control this fungus, or any European chestnut cultivar tolerant or resistant to the disease. In recent years, research on chestnut blight control has focused on the development of biological methods. Cryphonectria parasitica has two types of strains: virulent, causing severe lesions to the tree, and hypovirulent, which cause hardly any damage just because they are carriers of a virus that attenuates virulence. Field application, on affected chestnuts, of hypovirulent strains, which can transmit the virus to the virulent ones, is by far the only prospect for reducing and/or minimize the damage that this pathogen causes. The success of this biological control method for chestnut blight requires prior knowledge of the population structure of Cryphonectria parasitica (number and distribution of vegetative compatibility and sexual types) and hypovirulent isolates compatible with the virulent strains that are dominant in an affected area.

Keywords: Castanea sativa, dsRNA virus, vegetative compatibility, mating types

\section{Introducción}

La enfermedad del cancro, causada por el ascomiceto Cryphonectria parasitica (Murrill) M.E. Barr (sinónimo Endothia parasitica), originario del este de Asia, está considerada como el problema fitosanitario más grave del castaño a nivel mundial. La forma virulenta del hongo causa lesiones necrosadas en la corteza, que van aumentando progresivamente de tamaño hasta originar la muerte de ramas y troncos.

No todas las especies del género Castanea presentan el mismo grado de susceptibilidad al ataque de $C$. parasitica. Las más sensibles son Castanea dentata (castaño americano) y Castanea sativa (castaño común o castaño 
europeo). Las menos susceptibles son las especies asiáticas Castanea mollissima (castaño chino) y Castanea crenata (castaño japonés), debido a que, tras años de coexistencia y evolución con $C$. parasitica, han adquirido una tolerancia natural a este patógeno (Huang et al., 1998). Cryphonectria parasitica también puede infectar a otras frondosas, como Acer spp., Alnus cordata, Carpinus betulus, Carya ovata, Castanopsis spp., Ostrya carpinifolia, Quercus pubescens, Q. petraea, Q. frainetto, Q. ilex y Rhus typhina (Dallavalle y Zambonelli, 1999; EPPO, 2005).

El primer caso de la enfermedad se detectó en 1904 en castaños del parque zoológico del Bronx, en Nueva York (Merkel, 1905). Probablemente el hongo llegó allí desde Asia y se extendió al resto del mundo a través de material de vivero o árboles infectados (Milgroom et al., 1996). En Estados Unidos, la enfermedad se propagó rápidamente, de tal forma que en 1950 había destruido la práctica totalidad de los castaños americanos dentro de su hábitat natural, provocando un gran desastre ecológico. Se cree que C. parasitica causó la muerte de 3.500 millones de árboles en un periodo de 40 años (Roane et al., 1986). Como consecuencia, a día de hoy el castaño americano ha dejado de ser la especie dominante en los bosques de Norte América (Chaloux, 2000).

En Europa el cancro se detectó por primera vez en 1938 en el norte de Italia, en un bosque cercano a Génova (Biraghi, 1946). En 30 años se extendió rápidamente por Italia (Roane et al., 1986), y desde allí a países cercanos. En 1967, C. parasitica estaba presente en la mayoría de las zonas de castaños de Francia, Suiza, Turquía, España, Grecia y Hungría (Griffin, 1986).

La incidencia de cancro es hoy particularmente preocupante en los países con mayor superficie de castaño: Francia e Italia (Robin et al., 2000; Turchetti et al., 2008), que acumulan el $79,3 \%$ de la superficie total europea (2,03 millones de hectáreas de un total de 2,56), y Suiza, España y Portugal (Cortesi et al., 1998; Valdezate et al., 2001; Homs et al., 2002; Bragança et al., 2007; Aguín et al., 2008; Montenegro et al., 2008), que abarcan un 9,7\% (248.320 ha) (Conedera et al., 2004). También está presente en muchos otros países europeos con poca superficie de castaño, como Alemania, Grecia, Turquía, Macedonia, Bosnia, Croacia, Hungría, Rumanía, Eslovaquia, Bélgica y Holanda (EPPO, 2011). Las graves consecuencias del cancro para la persistencia de los castaños europeos justifica que $C$. parasitica esté considerado un patógeno de cuarentena incluido en la lista A2 de la Organización Europea y Mediterránea para la Protección de las Plantas (EPPO, 2011).

En España, se cree que $C$. parasitica se introdujo en la primera mitad del siglo XX. Rodríguez Sardiña identificó en 1940 a Endothiella gyrosa Sacc. como causante de una sintomatología similar al cancro en una plantación de castaños asiáticos en Córgoma de Valdeorras (Galicia); sin embargo, la primera vez que se cita a Endothia parasitica como responsable del cancro del castaño europeo es en Vizcaya en 1947 (Elorrieta, 1949).

\section{Características del cancro del castaño}

\section{Proceso de infección por Cryphonectria parasitica}

\section{Entrada y colonización de la corteza}

El proceso de infección de Cryphonectria parasitica comienza al penetrar las esporas (conidios o ascosporas) en la corteza del castaño a través de aberturas naturales o heridas provocadas por el hombre, insectos, etc. (EPPO, 2005). El patógeno ataca principalmente al tronco y ramas del árbol (Goidanich, 1982).

\section{Formación de cancros}

Generalmente, los primeros síntomas de la enfermedad suelen aparecer un mes después de comenzar la infección por C. parasitica, siendo el más característico la aparición de lesiones en la corteza, que se denominan cancros. En un punto del tronco o de una rama aparecen unas zonas pardoamarillentas, de contorno irregular, y se produce un enrojecimiento y ligera hinchazón de la corteza, que posteriormente se resquebraja longitudinalmente. Adherido a la parte interna de la corteza, se observa el micelio blanco del patógeno con forma de abanico (Goidanich, 1982). El micelio puede sobrevivir hasta 10 meses en corteza seca (Hepting, 1974). En la zona de la corteza afectada, se forman pequeñas pústulas de color amarillo-anaranjado, que son los cuerpos de fructificación (picnidios y peritecios) del hongo.

Cryphonectria parasitica presenta una fase sexual y otra asexual. En la asexual, el hongo forma picnidios globosos, de color de amarillo a anaranjado, variables en tamaño (100-300 $\mu \mathrm{m}$ de diámetro) y con un ostiolo que emite esporas. Los picnidios pueden aparecer aislados 0 agregados, formando grupos de más de $1 \mathrm{~mm}$ de diámetro (EPPO, 2005). En condiciones de humedad elevada, los picnidios liberan esporas asexuales (conidios) englobadas en un material viscoso, que forma cadenas retorcidas de color amarillo denominadas cirros. La emisión de conidios se produce de forma más abundante en primavera y en otoño.

Los conidióforos presentan una longitud máxima de $60 \mu \mathrm{m}$ de largo y 1,5 $\mu \mathrm{m}$ de ancho; son ramificados, septados e hialinos. Los conidios tienen un tamaño de aproximadamente 3,6 x 1,8 $\mu \mathrm{m}$; son unicelulares, hialinos, aseptados, de forma elipsoidal a baciliforme y en ocasiones ligeramente curvados.

En la fase sexual, el hongo forma peritecios globosos, de 300 a $400 \mu \mathrm{m}$ de diámetro, apareciendo en grupos de 5 a 50 unidades. Dentro de los peritecios se forman las ascas, que contienen las ascosporas. Las ascas tienen un tamaño aproximado de 32-55 $\mu \mathrm{m}$ de largo por 7-8,5 $\mu \mathrm{m}$ de ancho, y presentan paredes finas. Cada asca contiene 8 ascosporas dispuestas en dos filas. Las ascosporas son elípticas, 
bicelulares, hialinas, uniseptadas y con un tamaño medio de 8,6 x 4,5 um (Sivanesan y Holliday, 1981; EPPO, 2005). Los peritecios liberan ascas, que pueden ser expulsadas a varios centímetros de distancia (Goidanich, 1982).

En algunos casos, las fases asexual y sexual pueden coexistir, observándose picnidios y peritecios simultáneamente (Mansilla et al., 2000). En campo, los peritecios son menos frecuentes que los picnidios (Heiniger y Rigling, 1994). In vitro, C. parasitica no forma peritecios, completando únicamente la fase asexual (EPPO, 2005).

En la rama y/o tronco invadido, C. parasitica infecta el cambium y el xilema, interrumpiendo el suministro de savia, provocando el marchitamiento de hojas y ramas, un decaimiento general del árbol, y la formación de brotes epicórmicos justo debajo del cancro. La parte afectada de la planta sufre un proceso más o menos rápido de necrosis, que se detecta por la aparición de ramas secas a partir de la lesión (Milgroom y Cortesi, 2004). Progresivamente los cancros van aumentando de tamaño y rodeando a ramas y troncos. Cuando esto sucede, los tejidos vegetales situados por encima de la lesión terminan por morir. En la figura 1 se muestra un tronco de castaño europeo afectado por Cryphonectria parasitica.

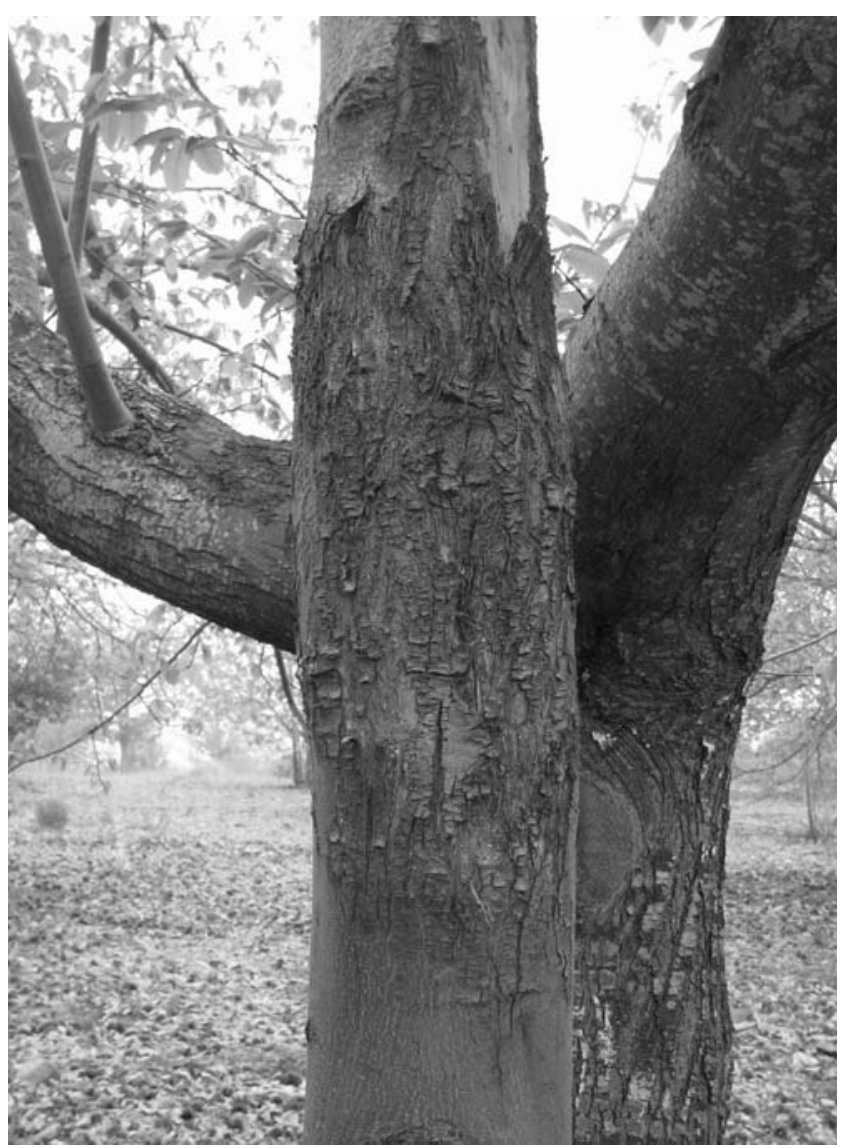

Figura 1.- Tronco de castaño europeo afectado por Cryphonectria parasitica en el que se observan síntomas de cancro, con grietas longitudinales y descortezamiento

\section{Transmisión del cancro}

El principal agente de propagación de $C$. parasitica es el viento, que puede transportar las ascosporas a gran distancia. Los conidios, sin embargo, aunque también dan lugar a la enfermedad, no presentan dispersión aérea, ya que están englobados en una sustancia viscosa que se lo impide, y son transportados por la lluvia o en las patas, plumas, pelo, etc., de pequeños animales e insectos (Goidanich, 1982; Mansilla et al., 2000).

No se conoce bien el papel que pueden jugar los insectos en la propagación de este hongo, pero los escasos estudios realizados indican que numerosas especies pueden ser agentes vectores. Se ha demostrado que hormigas y ácaros pueden transportar propágulos viables de este patógeno (Anagnostakis, 1982; Wendt et al., 1983). Russin et al. (1984), en Estados Unidos, encontraron 69 especies de insectos (la mayoría incluidas en el orden Coleoptera) que los transportaban.

El hombre ha contribuido de forma importante a la propagación del cancro, sobre todo mediante el transporte de material infectado a zonas libres de la enfermedad y la utilización de herramientas contaminadas y sin desinfectar en labores de poda.

\section{Biología de las poblaciones de Cryphonectria parasitica}

\section{Incompatibilidad vegetativa}

En los hongos filamentosos, como C. parasitica, existe la posibilidad de que se produzcan anastomosis hifales entre micelios de dos cepas diferentes, dando lugar a heterocariontes cuando los dos núcleos genéticamente diferentes coexisten en un mismo citoplasma (Glass et al., 2000). La formación de heterocariontes puede tener aspectos beneficiosos, ya que favorece el intercambio genético y el heterocarionte dispone de más biomasa para utilizar los recursos disponibles (Glass et al., 2000), pero también perjudiciales, ya que aumenta el riesgo de transmisión de elementos citoplasmáticos infecciosos, como virus de ARNbc, plásmidos, transposones y orgánulos debilitados (Saupe, 2000).

A pesar de los posibles beneficios, estos hongos presentan un sistema de incompatibilidad vegetativa, que permite a un individuo diferenciar sus propias células de las de otros, y restringe la formación de heterocariontes. Este sistema está controlado genéticamente por múltiples genes dispersos por el genoma, denominados genes o loci vic. La mayoría de los loci vic presentan 2 alelos, aunque en algunas especies se ha observado la existencia de alelos múltiples (Milgroom y Cortesi, 1999).

Dos individuos son incompatibles cuando presentan alelos diferentes en uno o más genes vic. Las uniones entre ellos son transitorias y las células fusionadas mueren (Cortesi et al., 2001). Cuando los individuos poseen alelos idénticos en 
todos los loci vic, se forma un heterocarionte estable, que indica que los individuos son compatibles vegetativamente y pertenecen al mismo tipo de compatibilidad vegetativa (tipo vc). La clasificación de los individuos en grupos de

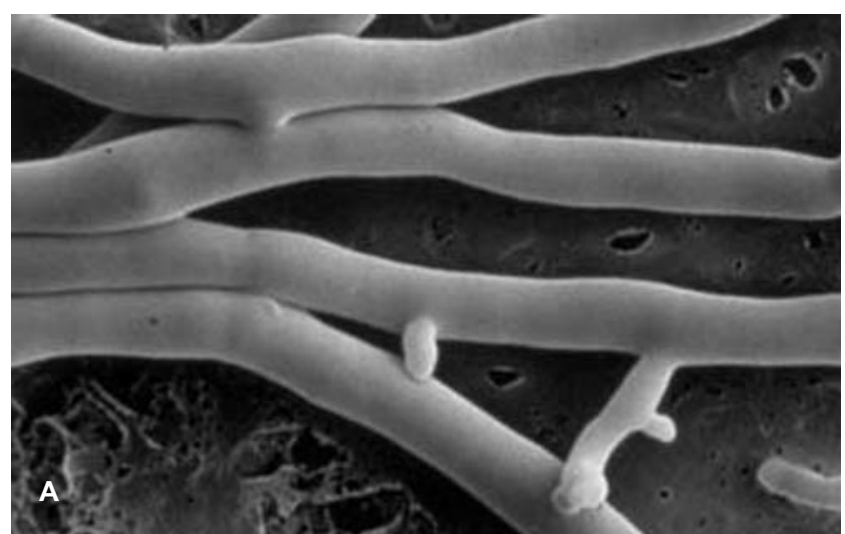

compatibilidad vegetativa es muy útil para estudiar la diversidad genética de las poblaciones. En la figura 2 se muestran contactos entre hifas de aislados de C. parasitica compatibles e incompatibles.

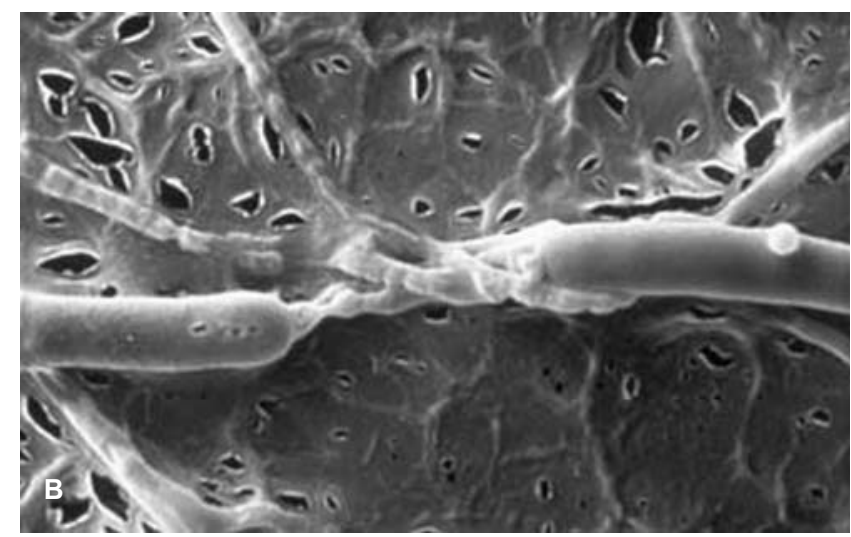

Figura 2.- Reacciones de compatibilidad entre aislados de Cryphonectria parasitica (Heiniger, 1997). A: reacción compatible, donde se observa fusión de hifas. B: reacción incompatible, con estrangulamiento de hifas

Estudios realizados en Europa en la década de los 90 llevaron a considerar que el sistema de incompatibilidad vegetativa en $C$. parasitica estaba controlado por 6 genes vic no ligados, con dos alelos cada uno (Cortesi y Milgroom, 1998). Los loci vic se denominaron vic1, vic2 vic3, vic4, vic6 y vic7, los cuatro primeros descritos por Anagnostakis (1982) y Huber (1996) y los otros dos por Cortesi y Milgroom (1998). Estos autores estudiaron también el loci vic5, pero no pudieron demostrar que estuviera implicado en el control de la incompatibilidad, por lo que no fue considerado en posteriores estudios de incompatibilidad vegetativa en $C$. parasitica. Considerando que con los seis loci vic son posibles 64 genotipos $\left(2^{6}=64\right)$, Cortesi y Milgroom (1998) establecieron 64 tipos de compatibilidad vegetativa de $C$. parasitica en Europa, que están considerados como tipos vc de referencia europeos y denominados EU1-EU64.

Pocos años después, Robin et al. (2000) encontraron 10 genotipos vc adicionales en poblaciones de C. parasitica de Francia, que se reconocieron como tipos europeos de referencia con los códigos EU65-EU74, lo que indicaba que el número de loci vic inicialmente considerado tenía que ser al menos de siete. A esta hipótesis contribuyen los trabajos de Bragança et al. (2007), en poblaciones de C. parasitica en Portugal, y Aguín et al. (2008) y Montenegro et al. (2008), en poblaciones de España, que comunicaron respectivamente dos y siete tipos vc diferentes de los 74 europeos de referencia, y coincide con la hipótesis pionera de Wang et al. (1991) que ya apuntaban que la compatibilidad vegetativa en $C$. parasitica debía de estar controlada por al menos ocho loci vic para poder explicar los 131 tipos vc que estos autores encontraron en China.

Aunque el número posible de tipos vc es alto, la diversidad de grupos vc en las poblaciones europeas de C. parasitica es baja, con índices de Shannon generalmente inferiores a 2 (Garbelotto et al., 1992; Cortesi et al., 1998; Robin et al.,
2000; Trestic et al., 2001; Sotirovski et al., 2004; Perlerou y Diamandis, 2006; Bragança et al., 2007; Montenegro et al., 2008; González-Varela et al., 2011), mientras que la diversidad de grupos vc es muy alta en poblaciones de Estados Unidos, con índices de Shannon de 2,7-2,8 (Anagnostakis et al., 1986; Anagnostakis y Kranz, 1987) y sobre todo de países asiáticos como China y Japón, de donde es nativa $C$. parasitica, con índices de 2,4-3,3 y 1,93,3 , respectivamente (Liu y Milgroom, 2007). La diversidad de tipos de compatibilidad vegetativa de $C$. parasitica se ha estudiado de forma extensa en Europa (Tabla 1). Los tipos vc de C. parasitica más frecuentes son EU-1, EU-2 y EU-12, que podrían haberse introducido en los distintos países europeos a partir de un origen común. Sin embargo, aunque la caracterización de los tipos vc es útil para estimar la diversidad de este patógeno, se ha descubierto, utilizando marcadores moleculares, que existe bastante diversidad dentro de los tipos vc en algunas poblaciones europeas (Breuillin et al., 2006). Por ello estudios recientes sugieren que, para mejorar el análisis de la estructura de las poblaciones y averiguar la historia de introducción y dispersión de este patógeno, es necesario la utilización de marcadores moleculares neutros y codominantes, como los microsatélites (Milgroom et al., 2008).

Se cree que el número de tipos vc presentes tiende a incrementarse con el tiempo mediante recombinación sexual entre las cepas existentes, por la introducción de nuevos grupos o por mutación genética (Adamčíková et al., 2006). Por este motivo algunos autores sugieren que la diversidad de tipos vc en una zona determinada es mayor cuanto más tiempo haya transcurrido desde la introducción de la enfermedad (Robin y Heiniger, 2001).

En España, los tipos y la dominancia de tipos vc son muy diferentes en las principales zonas de castaño, incluso entre zonas geográficas limítrofes (Tabla 2). En Cataluña (Trestic 
et al., 2001), las poblaciones de C. parasitica están dominadas por el tipo vc EU-2, en Navarra por EU-66, en Asturias y Castilla y León (Montenegro et al., 2008; González-Varela et al., 2011) por el tipo EU-1, y en Galicia es dominante E1, un tipo vc no incluido en el listado de tipos europeos de referencia, si bien también está presente el tipo EU-1 (Aguín et al., 2008; Montenegro et al., 2008). Estas diferencias en los tipos vc dominantes entre poblaciones del patógeno en España sugieren que $C$. parasitica se ha establecido independientemente en muchas áreas afectadas. Es posible, sin embargo, que la línea clonal EU1 tuviera una única introducción en el noroeste de España, lo que tendrá que verificarse mediante marcadores microsatélites en el futuro.

\begin{tabular}{lll}
\hline Pais & Tipos vc EU más abundantes & Referencias \\
\hline Alemania & EU-2 & Seeman et al. (2001) \\
Bosnia-Herzegovina & EU-1, EU-2, EU-12, EU-13 & Trestic et al. (2001) \\
Croacia & EU-1 & Krstin et al. (2008) \\
Eslovaquia & EU-12 & Adamč?kov? et al. (2006) \\
Eslovenia & EU-13 & Krstin et al. (2011) \\
España & EU-1, EU-2 & Colinas y Uscuplic (1998), Montenegro et al. \\
Francia & EU-1, EU-2, EU-5, EU-33 & (2008), González-Varela et al. (2011) \\
Grecia & EU-12 & Robin et al. (2000) \\
Hungria & EU-1, EU-6, EU-12, EU-13 & Riamandis y Perlerou (2006) \\
Italia & EU-1, EU-2, EU-5, EU-10, EU-12 & Cortesi et al. (1996, 1998) \\
Macedonia & EU-12 & Sotirovski et al. (2004) \\
Portugal & EU-11 & Bragança et al. (2007) \\
Rumanía & EU-12 & Radócz (2001) \\
Suiza & EU-1, EU-2, EU-5 & Cortesi et al. (1998) \\
Turquía & EU-1 & Çeliker y Onoğur (2001), Akilli et al. (2009) \\
Ucrania & EU-12 & Radócz (2001) \\
\hline & &
\end{tabular}

Tabla 1.- Tipos vc más abundantes en las poblaciones europeas de Cryphonectria parasitica

\begin{tabular}{llll}
\hline $\begin{array}{l}\text { Comunidad } \\
\text { Autónoma }\end{array}$ & $\begin{array}{l}\text { Tipo vc } \\
\text { dominante }\end{array}$ & $\begin{array}{l}\text { Otros tipos vc } \\
\text { encontrados }\end{array}$ & Referencias \\
\hline Galicia & E1 & EU-1, EU-9, EU-66, EU-72, & $\begin{array}{l}\text { Aguín et al. (2008), } \\
\text { Montenegro et al. (2008) }\end{array}$ \\
$\begin{array}{l}\text { A Coruña } \\
\text { Lugo }\end{array}$ & EU-1 & E2, E5, E6, E7 & \\
$\begin{array}{l}\text { Ourense } \\
\text { Pontevedra }\end{array}$ & E1 & E2, E5, E7 & \\
Asturias & EU-1 & EU-1, EU66, E2, E5, E6, E7 & \\
Cataluña & EU-2 & EU-3, EU-13 & González-Varela et al. (2011) \\
Castilla y León & EU-1 & EU-1, EU-5, EU-12, EU-36, & Colinas y Uscuplic (1998), \\
Navarra & EU-66 & EU-66, EU-66, EU-72 & Trestic et al. (2001) \\
\hline
\end{tabular}

Tabla 2.- Tipos vc en las poblaciones españolas de Cryphonectria parasitica

\section{Reproducción sexual}

En C. parasitica, al igual que en la mayoría de los ascomicetos filamentosos heterotálicos, los genes responsables del apareamiento sexual están localizados en un único locus, denominado MAT (del inglés mating type), del que existen dos idiomorfos, MAT-1 y MAT-2 (Coppin et al., 1997; Kronstad y Staben, 1997; Marra y Milgroom, 1999). Se habla de un sistema de apareamiento bipolar, ya que solamente ocurre entre individuos que poseen idiomorfos o alelos MAT diferentes (Coppin et al., 1997; Kronstad y Staben, 1997).

En el estudio de las poblaciones de C. parasitica, es necesario averiguar la incidencia de cada alelo MAT para poder conocer la probabilidad de reproducción sexual entre las distintas cepas.

Muchas poblaciones europeas de C. parasitica están dominadas por uno de los tipos MAT, como sucede en Suiza (Hoegger et al., 2000), Macedonia y Grecia (Sotirovski et al., 2004) y España (Aguín et al., 2008; Montenegro et al., 2008; González-Varela et al., 2011), donde el tipo MAT-1 es más abundante que el MAT-2. Por el contrario en Portugal, Bragança et al. (2007) encontraron ratios de tipos MAT próximos a 1:1. La proporción de tipos MAT es un reflejo de la importancia que la reproducción sexual presenta en una población de $C$. parasitica. En poblaciones donde la forma de reproducción predominante es la sexual cabría esperar que los dos tipos MAT estuviesen presentes en una proporción 1:1, mientras que en las poblaciones en las que 
un tipo MAT predomina sobre el otro, la ratio se desvía de la proporción 1:1 y el tipo de reproducción es fundamentalmente asexual (Anagnostakis, 1988).

\section{La hipovirulencia}

Existen diferentes hongos patógenos de plantas, entre los que se encuentra $C$. parasitica, que presentan dos tipos de cepas: unas virulentas, que llevan rápidamente a la aparición de síntomas y posterior muerte de la especie vegetal afectada, y otras menos agresivas, que determinan menos síntomas, menos daños y no causan la muerte del hospedador. Esta atenuación de la virulencia que caracteriza a algunas cepas fúngicas se conoce como hipovirulencia y se debe a la infección del hongo patógeno por un micovirus (Nuss, 2005).

Los micovirus se encuentran en los principales grupos taxonómicos de hongos, infectando a sus hospedadores sin que la mayoría produzca ningún cambio en su fenotipo (Buck, 1986). Solo unos pocos micovirus pueden alterar la virulencia de hongos que son patógenos de plantas. Todos los micovirus asociados con el fenómeno de la hipovirulencia poseen genomas de ARN, bien monocatenario (ARNmc) ó bicatenario (ARNbc).

Inicialmente el daño ocasionado en el castaño europeo por C. parasitica fue importante, provocando una elevada mortalidad (Heiniger y Rigling, 1994). Sin embargo, en 1950, en Italia, solamente 12 años después del descubrimiento del cancro del castaño en Europa, Biraghi (1953) observó que la dispersión del hongo era más lenta en Europa que en Norte América, y había indicios de que algunos castaños europeos afectados se estaban recuperando del cancro, como lo indicaba el hecho de que muchos de los nuevos brotes de castaños afectados sobrevivían más de los 4 ó 5 años habituales y que muchos de los cancros cicatrizaban espontáneamente, quedando el hongo relegado a la capa más superficial de la corteza. Grente (1965) consiguió muestras de estos cancros cicatrizados de Italia y obtuvo aislados de C. parasitica que inoculó en lesiones de castaño causadas por cepas virulentas, observando posteriormente la remisión de los cancros. Hizo después un reaislamiento a partir de los cancros cicatrizados y encontró que la mayoría de los nuevos aislados eran hipovirulentos, sugiriendo que la aplicación de aislados hipovirulentos en los cancros causados por otros virulentos había dado lugar a anastomosis hifales entre ellos y a la conversión de cepas virulentas en hipovirulentas (Scheffer, 1997).

Las cepas hipovirulentas en campo son difíciles de detectar. En general tienen un crecimiento más lento que las virulentas y causan cancros superficiales, que apenas ocasionan daños en el árbol (Heiniger y Rigling, 1994). En estos cancros, la producción de picnidios es escasa y la reproducción sexual aparece prácticamente inhibida (Elliston, 1985), el micelio queda restringido a las capas externas de la corteza y con frecuencia no presenta la forma típica de abanico de los cancros virulentos (Heiniger y Rigling, 1994). El crecimiento en cultivo también es más lento que el de las cepas virulentas y la apariencia de la colonia suele ser lobulada y con escaso micelio aéreo, a diferencia de las virulentas que presentan un crecimiento organizado (Elliston, 1985).

Hasta hace pocos años, la diferenciación in vitro entre cepas hipovirulentas y virulentas se basaba en características morfológicas de las colonias en cultivo, observando la coloración del micelio, crecimiento, textura, presencia de cuerpos de fructificación, etc., lo que llevaba en la mayoría de los casos a resultados confusos. Sin embargo, la puesta a punto de técnicas moleculares para detectar y caracterizar los virus responsables de la hipovirulencia ha facilitado enormemente la determinación rápida y fiable de las cepas atenuadas. Las técnicas moleculares que se utilizan actualmente para la detección de hipovirulencia en $C$. parasitica incluyen el aislamiento del ARNbc del micovirus, la reversotranscripción del ARN vírico a ADNc, la amplificación del ADN vírico mediante PCR y el análisis de polimorfismos de fragmentos de restricción (RFLP) del ADN vírico amplificado (Allemann et al., 1999).

Inicialmente se sugirió que la hipovirulencia en C. parasitica se debía a la presencia de unos determinantes citoplasmáticos transmisibles (Grente y Sauret, 1969). Day et al. (1977) identificaron a estos determinantes como moléculas de ARNbc y postularon que tenían un origen vírico, a pesar de que no consiguieron aislar partículas víricas a partir de las cepas hipovirulentas. En la década de los 80 y comienzos de los 90 , el análisis del ARNbc de aislados hipovirulentos de C. parasitica indicó la existencia de diferente número y concentración de múltiples especies de ARNbc (Anagnostakis y Day, 1979; Dodds, 1980; Kuhlman et al., 1984; Elliston, 1985; Paul y Fulbright, 1988), que se clasificaron en función de su tamaño en $L$ (long)ARNbc (aprox. $12 \mathrm{kpb}$ ), M (medium)-ARNbc (aprox. 8-10 $\mathrm{kpb}$ ) y $\mathrm{S}$ (short)-ARNbc (aprox. 0,6-1,7 kpb) (Hiremath et al., 1986; Shapira et al., 1991a). Las especies M y S son formas truncadas del L-ARNbc, mantenidas en la cepa hipovirulenta como consecuencia de la retención de dominios de secuencia esenciales para la replicación (Shapira et al., 1991a). Las especies M y S de ARNbc pueden variar en número y tamaño durante el subcultivo, sin embargo la forma $L$ permanece estable en tamaño y concentración (Shapira et al., 1991a).

Finalmente se demostró el origen vírico del ARNbc de los aislados hipovirulentos (Shapira et al., 1991b; Choi y Nuss, 1992). Los estudios realizados en los últimos 15 años han demostrado la gran diversidad de especies víricas presentes en aislados de C. parasitica. Los virus descritos pertenecen a cuatro familias: Reoviridae, Narnaviridae, Partitiviridae e Hypoviridae (Hillman y Suzuki, 2004).

Existe muy poca información respecto a los virus de las familias Reoviridae, Naranaviridae y Partitiviridae, ya que se han encontrado en muy pocas cepas de $C$. parasitica de Norte América y China. En general, producen poco o ningún efecto sobre la esporulación y pigmentación de las colonias fúngicas y muestran grandes diferencias en su acción hipovirulenta: desde moderada a severa (Polashock y Hillman, 1994; Peever et al., 1998; Hillman et al., 2004).

En la familia Hypoviridae se incluyen 4 especies, que presentan diferencias en cuanto a la estructura del genoma, 
la relación entre secuencias y los síntomas causados al hongo hospedador, denominadas Cryphonectria hypovirus 1 (CHV1) (Shapira et al., 1991b), CHV2 (Hillmann et al., 1994), CHV3 (Smart et al., 1999) y CHV4 (Linder-Basso et al., 2005). Los hipovirus se localizan en el citoplasma del hongo y no presentan fase extracelular, ni poseen una verdadera cápside (figura 3 ).

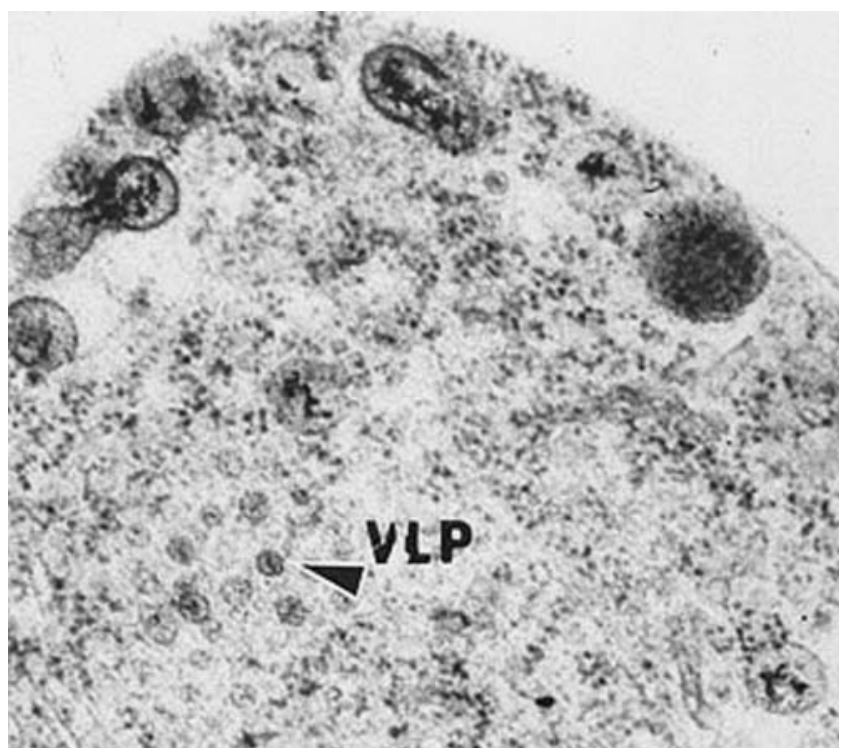

Figura 3.- Hifa de un aislado hipovirulento de Cryphonectria parasitica en la que se observan partículas de Cryphonectria hypovirus (VLP, virus like particles) (Anagnostakis, 2000)

A partir de hifas de C. parasitica han podido aislarse vesículas de 50 a $80 \mathrm{~nm}$ que contienen el genoma del virus, formado por ARNbc lineal no fragmentado con un tamaño de entre 10 y 12 kpb (Hillmann et al., 1995; Nuss, 2005). Los hipovirus alteran el fenotipo del hongo mediante la modificación de la expresión de genes fúngicos o la transcripción de proteínas e interfiriendo en el desarrollo de las rutas metabólicas y de las cascadas de señalización celular (Zhang et al., 1998; Parsley et al., 2002; Allen et al., 2003; Allen y Nuss, 2004), como la ruta de señalización de las proteínas G (Dawe y Nuss, 2001). Se ha visto que esta ruta desempeña un papel fundamental en la virulencia de organismos como Fusarium o Magnaporthe (Lengeler et al., 2000).

Se ha estimado que alrededor de un $20 \%$ de los genes de C. parasitica están afectados por la infección de CHV1, bien por un descenso o bien por un aumento en su expresión (Kang et al., 2000). Entre los genes cuya expresión se ve disminuida por los hipovirus están los que codifican actividades enzimáticas, como el de la lacasa, gen lac-1, y el de la celobiohidrolasa, cbh-1 (Rigling et al., 1989; Wang y Nuss, 1995). También la expresión del gen de la criparina, crp-1, proteína componente de la pared celular, está reducida (Kazmierczak et al., 1996).

Cryphonectria hypovirus 1 (CHV1) es la única especie presente en Europa, y también se ha descrito en China y
Japón (Peever et al., 1998). El tamaño de su genoma es de 12.712 pb. En Europa, CHV1 presenta una gran variabilidad. Allemann et al. (1999), mediante la caracterización molecular de 72 aislados hipovirulentos europeos, obtuvieron 5 subtipos: un subtipo italiano (CHV1I), que está ampliamente distribuido por las áreas afectadas de Europa, dos subtipos franceses (CHV1-F1 y CHV1-F2), un subtipo español (CHV1-E) y un subtipo alemán (CHV1D).

En España, todas las cepas hipovirulentas que se han detectado hasta la fecha portaban CHV1, identificándose los siguientes subtipos en las siguientes localizaciones: CHV1-E en un aislado de Navarra (Allemann et al., 1999) y cuatro de Galicia (Aguín et al., 2008), CHV1-I en un aislado de Cataluña (Homs et al., 2002) y CHV1-F1 en quince aislados de Castilla y León (Montenegro et al., 2008). También en Francia se han encontrado tres subtipos de CHV1 (I, F1 y F2) (Allemann et al., 1999). La diversidad de subtipos de CHV1 en España y Francia contrasta con lo encontrado en otros países europeos, como Bosnia, Croacia, Grecía, Hungría, Italia y Suiza, donde solo se ha detectado el subtipo italiano.

Actualmente existen dos aislados considerados como virus CHV1 tipo: CHV1-EP713, aislado en Francia en 1966 (Shapira et al., 1991b) y perteneciente al subtipo F1 (Allemann et al., 1999), y CHV1-Euro7, aislado en Italia en 1977 (Chen y Nuss, 1999) e incluido en el subtipo I (Allemann et al., 1999). En cultivo, los aislados de C. parasitica infectados por CHV1-EP713 o por CHV1-Euro7 presentan menos pigmentación anaranjada y menor producción de conidios que las cepas del patógeno libres de virus.

La secuencia de los dos aislados CHV1 tipo es muy similar (homología de 87 a $93 \%$ a nivel nucleotídico y de 90 a $98 \%$ a nivel aminoacídico), pero los efectos en la esporulación, crecimiento y forma de colonias que producen en $C$. parasitica son diferentes (Chen y Nuss, 1999). Además, sobre el árbol, las cepas infectadas por el tipo CHV1-EP713 producen cancros pequeños y superficiales, con pocos o ningún cuerpo de fructificación, mientras que los cancros producidos por la cepa CHV1-Euro7 se expanden de manera agresiva en las fases iniciales pero, cuando se produce el callo de cicatrización en los márgenes de la infección, su crecimiento se detiene de manera abrupta. Debido a estas diferencias, las cepas CHV1-EP713 y CHV1-Euro7 se denominan de hipovirulencia severa y suave, respectivamente (Chen y Nuss, 1999).

Cryphonectria hypovirus 2 (CHV2) está presente en Norte América y China (Peever et al., 1998). Tiene como especie tipo a la cepa CHV2-NB58, originaria de New Jersey (Hillman et al., 1992). En cultivo los aislados de C. parasitica infectados por CHV2-NB58 muestran un micelio de color parduzco anaranjado, crecimiento lento y ausencia de micelio aéreo (Hillman et al., 1994).

Cryphonectria hypovirus 3 (CHV3) es originario de Grand Haven, en el estado de Michigan (Fulbright et al., 1983). La cepa CHV3-GH2 está considerada la especie tipo de este hipovirus (Nuss et al., 2006) y, cuando infecta a aislados de C. parasitica, produce reducción en la virulencia pero no 
reduce la conidiación, por lo que las colonias fúngicas presentan la típica pigmentación de color anaranjado de las cepas virulentas (Smart et al., 1999).

Cryphonectria hypovirus 4 (CHV4) es la especie más extendida en el este de Norte América (Peever et al., 1997). La especie tipo de este hipovirus es CHV4-SR2 (Nuss et al., 2006), que se diferencia de los demás hipovirus en que apenas produce efectos en la virulencia del hongo o en la morfología de las colonias (Enebak et al., 1994).

\section{Control biológico del cancro del castaño}

No existe ningún método eficaz cultural ni químico de control de $C$. parasitica, ni ningún cultivar de castaño europeo tolerante o resistente a la enfermedad (EPPO, 1997). En la práctica, la introducción y diseminación de la enfermedad en áreas libres de cancro se evita mediante medidas preventivas. La legislación europea prohíbe el transporte de castaños sintomáticos en su territorio, y establece la obligación de los productores de planta de poseer un pasaporte fitosanitario en caso de venta (EPPO, 2005).

En áreas de castaño ya afectadas, la propagación de $C$. parasitica se intenta limitar mediante la eliminación de árboles sintomáticos o ramas afectadas, la desinfección de herramientas y la destrucción de restos de poda mediante quemas (Mansilla et al., 2003). Generalmente estas medidas no surten el efecto deseado debido a la facilidad con la que el patógeno se propaga.

Dada la ineficacia de métodos culturales y/o químicos, hace más de 25 años que la investigación sobre el control del cancro se ha centrado en el desarrollo de métodos biológicos basados en la transferencia del hipovirus desde cepas hipovirulentas 0 atenuadas a las virulentas (Anagnostakis, 1982), siendo la biología de C. parasitica en cada zona afectada un factor clave para poder aplicar con éxito estas técnicas (Heiniger y Rigling, 1994; Milgroom y Cortesi, 2004).

Van Alfen et al. (1975) demostraron que la hipovirulencia se puede transmitir de unas células fúngicas a otras mediante anastomosis hifales (figura 4).

La transmisión de las moléculas de ARNbc de una cepa a otra le confiere al individuo receptor el fenotipo hipovirulento, en un fenómeno denominado conversión (Anagnostakis, 1977; Anagnostakis y Day, 1979). La frecuencia y la estabilidad de la transmisión entre distintas cepas están determinadas por los genes que controlan la compatibilidad vegetativa (Anagnostakis, 1977). La conversión de una cepa virulenta in vitro se ve favorecida si pertenece al mismo grupo de compatibilidad vegetativa que la cepa hipovirulenta, aunque ocasionalmente también puede producirse entre cepas de distinto tipo vc (Anagnostakis y Day, 1979). La transmisión del hipovirus está relacionada inversamente con el número de genes vic diferentes que presentan las cepas que anastomosan (Liu y Milgroom, 1996). En campo, sin embargo, es imprescindible que el inóculo hipovirulento sea compatible con las cepas virulentas; en caso contrario, además de reducirse la posibilidad de conversión, podría facilitarse la aparición de aislados con nuevos tipos de compatibilidad vegetativa (por recombinación genética del $A D N$ de aislados que establezcan reproducción sexual) y en consecuencia nuevos focos de infección (Trestic et al., 2001).

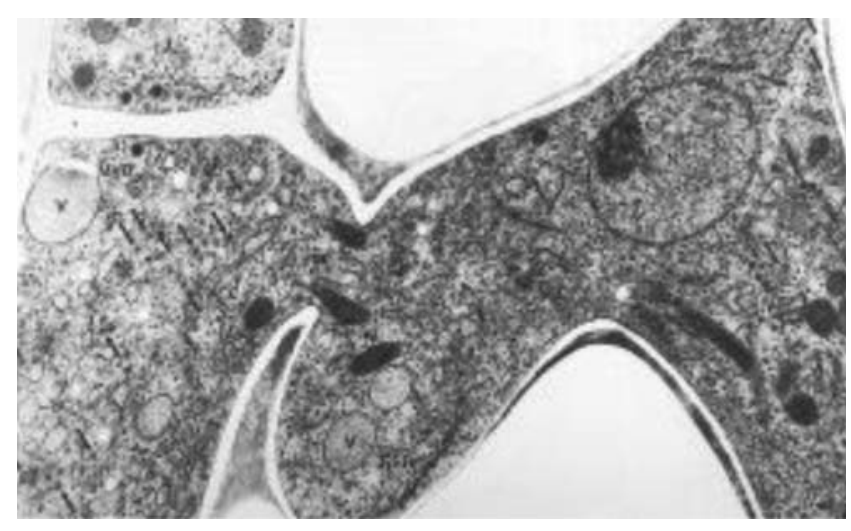

Figura 4.- Anastomosis establecida entre dos aislados de Cryphonectria parasitica pertenecientes al mismo tipo vc (Newhouse y McDonald, 1991). Las flechas señalan el paso de partículas de Cryphonectria hypovirus de un aislado a otro

La compatibilidad vegetativa es uno de los factores que afecta significativamente al éxito del control biológico de $C$. parasitica en campo (Heiniger y Rigling, 1994). El número de tipos vc en una zona afectada es de especial relevancia, ya que Cryphonectria hypovirus se transmite por anastomosis hifal o por conidios (Chen et al., 1993; Prospero et al., 1998), convirtiendo la cepa receptora al fenotipo hipovirulento (Anagnostakis y Day, 1979). Por ello, en el desarrollo de un programa de control biológico con cepas hipovirulentas, la existencia de un número bajo de grupos vc, como sucede en muchas poblaciones europeas de C. parasitica, favorecerá la dispersión del hipovirus y mejorará las posibilidades en el control de la enfermedad. Es el caso de algunas zonas de Francia o Italia, donde el número de tipos vc en las poblaciones del hongo es bajo y el biocontrol con cepas hipovirulentas ha resultado exitoso (Grente y Berthelay-Sauret, 1978; Bisiach et al., 1995). Sin embargo, en Norte América, donde la diversidad es muy elevada, los programas de control biológico para el cancro no han funcionado como se esperaba y los intentos de introducción de cepas hipovirulentas han fracasado (Anagnostakis y Kranz, 1987).

Para que un programa de control biológico con cepas hipovirulentas sea eficaz, además de conocer la diversidad de los tipos vc y de disponer de cepas hipovirulentas compatibles con las virulentas dominantes en una zona afectada, es necesario averiguar también cual es la distribución de los tipos de apareamiento o mating type (Hoegger et al., 2000). La reproducción sexual en C. parasitica es un obstáculo importante para la diseminación de la hipovirulencia, porque los hipovirus no se transmiten mediante esporas sexuales (ascosporas) (Chen et al., 1993) y porque la recombinación genética, como ya se comentó, puede generar nuevos tipos de compatibilidad vegetativa. 
El éxito del control biológico será más probable en poblaciones con reproducción predominantemente asexual (Milgroom y Cortesi, 2004). Al haber poca o ninguna reproducción sexual, la diseminación de la hipovirulencia en campo dependerá del número de tipos vc (Anagnostakis, 1977, 1988).

Por lo tanto, se puede concluir que la idea de controlar el cancro en Castanea sativa mediante métodos biológicos es un objetivo alcanzable si las poblaciones de $C$. parasitica reúnen características de baja diversidad de tipos vc, reproducción predominantemente asexual y presencia de cepas hipovirulentas compatibles.

\section{Bibliografía}

Adamčíková, K., Juhásová, G \& Kobza, M. (2006). Genetic diversity of Cryphonectria parasitica population in the Štiavnicko-Krupinská subpopulation in Slovakia. Plant Protection Science. 42: 119-124.

Aguín, O., Mata, M., Mansilla, J.P., Martín, A.B. \& Sierra, J.M. (2005). Distribución y diversidad de los tipos de compatibilidad vegetativa de Cryphonectria parasitica (Murrill) Barr en castaños de Castilla y León. Boletín de Sanidad Vegetal Plagas. 31: 287-297.

Aguín, O., Montenegro, D., Sainz, M.J., Hermida, M. \& Mansilla, J.P. (2008). Caracterización morfológica y molecular de las poblaciones de Cryphonectria parasitica en castaños de Galicia. Boletín de Sanidad Vegetal Plagas. 34: 581-594.

Akilli, S., Katircioğlu, Y.Z. \& Maden, S. (2009). Vegetative compatibility types of Cryphonectria parasitica, causal agent of chestnut blight, in the Black Sea region of Turkey. Forest Pathology. 39: 390-396.

Allemann, C., Hoegger, P., Heiniger, U. \& Rigling, D. (1999). Genetic variation of Cryphonectria hypoviruses (CHV1) in Europe, assessed using restriction fragment length polymorphism (RFLP) markers. Molecular Ecology. 8: 843854.

Allen, T.D. \& Nuss, D.L. (2004). Linkage between mitochondrial hypovirulence and viral hypovirulence in the chestnut blight fungus revealed by cDNA microarray analysis. Eukaryotic Cell. 3: 1227-1232.

Allen, T.D., Dawe, A.L. \& Nuss, D.L. (2003). Use of cDNA microarrays to monitor transcriptional responses of the chestnut blight fungus Cryphonectria parasitica to infection by virulence-attenuating hypoviruses. Eukaryotic Cell. 2: 1253-1265.

Anagnostakis, S.L. (1977). Vegetative incompatibility in Endothia parasitica. Experimental Mycology. 1: 306-316.

Anagnostakis, S.L. \& Day, P.R. (1979). Hypovirulence conversion in Endothia parasitica. Phytopathology. 69: 1226-1229.

Anagnostakis, S.L. (1982). Biological control of chestnut blight. Science 215: 466-471.
Anagnostakis, S.L. \& Kranz, J. (1987). Population dynamics of Cryphonectria parasitica in a mixed-hardwood forest in Connecticut. Phytopathology. 77: 751-754.

Anagnostakis, S.L. (1988). Cryphonectria parasitica: cause of chestnut blight. Advances in Plant Pathology. 6: 123-136.

Anagnostakis, S.L. (2000). Revitalization of the majestic chestnut: chestnut blight disease. Disponible en: http://www.apsnet.org/publications/apsnetfeatures/Pages/ch esnutBlight Disease.aspx. [20 Junio, 2011].

Biraghi, A. (1946). II cancro del castagno causato da Endothia parasitica. L'Italia Agricola. 7: 406.

Biraghi, A. (1953). Possible active resistance to Endothia parasitica in Castanea sativa. En: Reports to 11th Congress of the International Union of Forest Research Organizations. International Union of Forest Research Organizations, Roma. 643-645.

Bisiach, M., Cortesi, P., De Martino, A. \& Intropido, M. (1995). Biological control of chestnut blight fungus Cryphonectria parasitica. En: Proceedings of the International Congress "Microbial control agents in sustainable agriculture field experience, industrial production and registration". Saint Vincent (Aosta). 167.

Bragança, H., Simões, S., Onofre, N., Tenreiro, R. \& Rigling, D. (2007). Cryphonectria parasitica in Portugal: diversity of vegetative compatibility types, mating types and occurrence of hypovirulence. Forest Pathology. 37: 391-402.

Buck, K.W. (1986). Fungal virology-an overview. En: Buck K.W. (Ed.) Fungal Virology. CRC Press, Boca Raton. pp. 284.

Çeliker N.M., Onoğur E. (2001). Evaluation of hypovirulent isolates of Cryphonectria parasitica for biological control of chestnut blight in Turkey. Forest Snow and Landscape Research. 76: 378-382.

Chaloux, P.H. (2000). Virulence of Cryphonectria hypoviruses from previous release sites. Master Thesis. West Virginia University.

Chen, B. \& Nuss, D.L. (1999). Infectious cDNA clone of hypovirus CHV1-Euro7: a comparative virology approach to investigate virus-mediated hypovirulence of the chestnut blight fungus Cryphonectria parasitica. Journal of Virology. 73: $985-992$

Chen, G., Choi, G.H. \& Nuss, D.L. (1993). Mitotic stability and nuclear inheritance of integrated viral cDNA in engineering hypovirulent strains of the chestnut blight fungus. EMBO Journal. 12: 2991-2998.

Choi, G.H., y Nuss, D.L. (1992). Hypovirulence of chestnut blight fungus conferred by an infectious viral cDNA. Science. 257: 800-803.

Colinas, C. \& Uscuplic, M. (1998). Cryphonectria parasitica vegetative compatibility (v/c) groups in the northeast Spain. Acta Horticulturae. 494: 495-500. 
Conedera, M., Manetti, M.C, Giudici, F. \& Amorini, E. (2004). Distribution and economic potential of the Sweet chestnut (Castanea sativa Mill.) in Europe. Ecologia Mediterranea. 30: 179-193.

Coppin, E., Debuchy, R., Arnaise, S. \& Picard, M. (1997). Mating types and sexual development in filamentous ascomycetes. Microbiology and Molecular Biology Reviews. 61: $411-428$.

Cortesi, P., Milgroom, M.G. \& Bisiach, M. (1996). Distribution and diversity of vegetative compatibility types in subpopulations of Cryphonectria parasitica in Italy. Mycological Research100: 1087-1093.

Cortesi, P. \& Milgroom, M.G. (1998). Genetics of vegetative incompatibility in Cryphonectria parasitica. Applied and Environmental Microbiology. 64: 2988-2994.

Cortesi, P., Rigling, D. \& Heiniger, U. (1998). Comparison of vegetative compatibility types in Italian and Swiss subpopulations of Cryphonectria parasitica. European Journal of Forest Pathology. 28: 167-176.

Cortesi P., McCulloch, C.E., Song, H., Lin, H. \& Milgroom, M.G. (2001). Genetic control of horizontal virus transmission in the chestnut blight fungus, Cryphonectria parasitica. Genetics. 159: 107-18.

Dalavalle, E. \& Zambonelli, A. (1999). Epidemiological role of strains of Cryphonectria parasitica isolated from hosts other than chestnut. European Journal of Forest Pathology. 29: $97-102$.

Dawe, A.L. \& Nuss, D.L. (2001). Hypoviruses and chestnut blight: exploiting viruses to understand and modulate fungal pathogenesis. Annual Review of Genetics. 35: 1-29.

Diamandis, C. \& Perlerou, S. (2006). Identification and geographic distribution of vegetative compatibility types of Cryphonectria parasitica and occurrence of hypovirulence in Greece. Forest Pathology. 36: 413-421.

Day, P.R., Dodds, J.A., Elliston, J.E., Jaynes, R.A. \& Anagnostakis, S.L. (1977). Double-stranded RNA in Endothia parasitica. Phytophathology. 67: 1393-1396.

Dodds, J.A. (1980). Revised estimates of the molecular weights of dsRNA segments in hypovirulent strains of Endothia parasitica. Phytophatology. 70: 1217-1220.

Elliston, J.E. (1985). Characteristics of dsRNA-free and dsRNA-containing strains of Endothia parasitica in relation to hypovirulence. Phytophatology. 75: 151-158.

Elorrieta, J. (1949). El castaño en España. Ministerio de Agricultura, Instituto Forestal de Investigaciones y Experiencias (IFIE), Madrid.

Enebak, S.A., Macdonald, W.L. \& Hillman, B.I. (1994). Effect of dsRNA associated with isolates of Cryphonectria parasitica from the central Appalachians and their relatedness to other dsRNAs from North America and Europe. Phytophatology. 84: 528-534.

EPPO. (1997). Cryphonectria parasitica. Quarantine Pests for Europe, $2^{\mathrm{a}}$ ed., páginas. 729-732. CAB International, Wallingford, Reino Unido.
EPPO. (2005). Diagnostics. Cryphonectria parasitica. EPPO Bulletin. 35: 295-298.

EPPO. (2011). Distribution maps of quarantine pest for Europe: Cryphonectria parasitica. Annex II/ A2. Disponible en: http://www.eppo.org/QUARANTINE/listA2.htm [20 junio 2011].

Fulbright, D.W., Weidlich, W.H., Haufler, K.Z., Thomas, C.S. \& Paul, C.P. (1983). Chestnut blight and recovering American chestnut trees in Michigan. Canadian Journal of Botany. 61: 3144-3171.

Garbelotto, M., Frigimelica, G. \& Mutto-Accordi, S. (1992). Vegetative compatibility and conversion to hypovirulence among isolates of Cryphonectria parasitica from northern Italy. European Journal of Forest Pathology. 22: 337-348.

Glass, N.L., Jacobson, D.J. \& Shiu, P.K.T. (2000). The genetics of hyphal fusion and vegetative incompatibility in filamentous ascomycete fungi. Annual Reviews of Genetics. 34: 165-186.

Goidanich, G. (1982). Manuale di patología vegetal. Edizioni Agricole, Bologne.

González-Varela, G., González, A.J. \& Milgroom, M.J. (2011). Clonal population structure and introductions of the chestnut blight fungus, Cryphonectria parasitica, in Asturias, northern Spain. European Journal of Plant Pathology. 131: 67-79.

Grente, J. (1965). Les formes hypovirulentes d'Endothia parasitica et les espoirs de lutte contre le chancre du châtaignier. Comptes Rendus Hebdomadaire des Séances de l'Académie d'Agriculture de France. 51: 1033-1037.

Grente, J. \& Berthelay-Sauret, S. (1978). Biological control of chestnut blight in France. En: W. L. MacDonald et al. Eds.). Proceedings of the American Chestnut Symposium. Virginia University Books, Morgantown. 30-34.

Grente, J. \& Sauret, S. (1969). L'hypovirulence exclusive, phénomène original en pathologie végétale. Comptes Rendus Hebdomadaire des Séances de l'Académie d'Agriculture de France. 286: 2347-2350.

Griffin, G. (1986). Chestnut blight and its control. Horticultural Reviews. 8: 291-335.

Heiniger, U. (1997). Le chancre de l'écorce du châtaignier (Cryphonectria parasitica): symptômes et biologie. En: Notice pour le praticien. 22. $2^{\mathrm{a}}$ edición. Birsmendorf.

Heiniger,U. \& Rigling, D. (1994). Biological control of chestnut blight in Europe. Annual Reviews of Phytopathology. 32: 581-589.

Hepting, G.H. (1974). Death of the American chestnut. Journal of Forest History 18: 60-67.

Hillman, B.I. \& Suzuki, N. (2004). Viruses of the chestnut blight fungus, Cryphonectria parasitica. Advances in Virus Research. 63: 423-472. 
Hillman, B.I., Fulbright, D.W., Nuss, D.L. \& Van Alfen, N.K. (1995). Hypoviridae. En: F.A. Murphy (Ed.). Sixth Report of the International Committee for the Taxonomy of Viruses. Springer-Verlag, New York.

Hillman, B.I., Halpern, B.T. \& Brown, M.P. (1994). A viral dsRNA element of the chestnut blight fungus with a distinct genetic organization. Virology. 201: 241-250.

Hillman, B.I., Supyani, S., Kondo, H. \& Suzuki, N. (2004). A reovirus of the fungus Cryphonectria parasitica that is infectious as particles and related to the Coltivirus genus of animal pathogens. Journal of Virology. 78: 892-898.

Hillman, B.I., Tian, Y., Bedker, P.J. \& Brown, M.P. (1992). A North American hypovirulent isolate of the chestnut blight fungus with European isolate-related dsRNA. Journal of General Virology. 73: 681-686.

Hiremath, S., L'Hostis, B., Ghabrial, S.A. \& Rhoads, R.E. (1986). Terminal structure of hypovirulence-associated dsRNAs in the chestnut blight fungus Endothia parasitica. Nucleic Acids Research. 14: 9877-9896.

Hoegger, P.J., Rigling, D., Holdenrieder, O. \& Heiniger, U. (2000). Genetic structure of newly established populations of Cryphonectria parasitica. Mycological Research. 104: 1108-1116

Homs, G., Rodríguez, J., Rigling, D. \& Colinas, C. (2002). Caracterización de la población de Cryphonectria parasitica y detección de cepas hipovirulentas en 3 subpoblaciones de Cataluña. En: Montes para la Sociedad del Nuevo Milenio. III Congreso Forestal Español. Ed. Junta de Andalucia, Granada.

Huang, H., Dane, F. \& Kubisiak, T. (1998). Allozyme and RAPD analysis of the genetic diversity and geographic variation in wild populations of the American chestnut (Fagaceae). American Journal of Botany. 85 (7): 1013-1021.

Huber, D.H. (1996). Genetic analysis of vegetative incompatibility polymorphisms and horizontal transmission in the chestnut blight fungus Cryphonectria parasitica. $\mathrm{PhD}$ Thesis. Michigan State University, East Lansing, Michigan, USA.

Kang, H.S., Choi, J.W., Park, S.M., Cha, B., Yang, M.S. \& Kim, D.H. (2000). Ordered differential display from Cryphonectria parasitica. Plant Pathology Journal. 16: 142146.

Kazmierczak, P., Pfeiffer, P., Zhang, L. \& Van Alfen, N.K. (1996). Transcriptional repression of specific host genes by the mycovirus Cryphonectria hypovirus 1. Journal of Virology. 70: 1137-1142.

Kronstad, J.W. \& Staben, C. (1997). Mating type in filamentous fungi. Annual Review of Genetics. 31: 245-276.

Krstin, L., Novak-Agbaba, S., Rigling, D., Krajačić, M. \& Ćurković Perica, M. (2008). Chestnut blight fungus in Croatia: diversity of vegetative compatibility types, mating types and genetic variability of associated Cryphonectria hypovirus 1. Plant Pathology. 57: 1086-1096.
Krstin L., Novak-Agbaba S., Rigling D. \& Ćurković Perica M. (2011). Diversity of vegetative compatibility types and mating types of Cryphonectria parasitica in Slovenia and occurrence of Cryphonectria hypovirus 1. Plant Pathology. 60: $752-761$

Kuhlman, E.G., Bhattacharyya, H., Nash, B.L., Double, M.L. \& Macdonald, W.L. (1984). Identifying hypovirulent isolates of Cryphonectria parasitica with broad conversion capacity. Phytopathology. 74: 676-682.

Lengeler, K.B., Davidson, R.C., D'Souza, C., Harashima, T., Shen, W.C., Wang, P., Pan, X., Waugh, M. \& Heitman, J. (2000). Signal transduction cascades regulating fungal development and virulence. Microbiology and Molecular Biology Reviews. 64: 746-785.

Linder-Basso, D., Dynek, J.N. \& Hillman, B.I. (2005). Genome analysis of Cryphonectria hypovirus 4, the most common hypovirus species in North America. Virology. 337: 192-203.

Liu, Y.C. \& Milgroom, M.G. (1996). Correlation between hypovirus transmission and the number of vegetative incompatibility (vic) genes different among isolates from a natural population of Cryphonectria parasitica. Plant Pathology. 86: 79-86.

Liu, Y.C. \& Milgroom, M.G. (2007). High diversity of vegetative compatibility types in Cryphonectria parasitica in Japan and China. Mycologia. 99: 279-284.

Mansilla, J.P., Salinero, C., Pérez, R. \& Pintos, C. (2003). Problemas fitosanitarios de los robles y castaños de Galicia. Ed. Diputación Provincial de Pontevedra, Pontevedra.

Mansilla, J.P., Pintos, C. \& Salinero, M.C. (2000). Plagas y enfermedades del castaño en Galicia. Ed. Xunta de Galicia.

Marra, R.E. (1998). Selfing in the context of selfincompatibility: the mixed mating system of the fungus Cryphonectria parasitica. PhD Thesis. Cornell University, Ithaca, USA.

Marra, R.E. \& Milgroom, M.G. (1999). PCR amplification of the mating-type idiomorphs in Cryphonectria parasitica. Molecular Ecology. 8: 1947-1950.

Merkel, H.W. (1905). A deadly fungus on the American chestnut. 10th Annual Report. of the New York Zoological Society. 97-103.

Milgroom, M.G., Macdonald, W.L. \& Double, M.L. (1991). Spatial analysis of vegetative compatibility groups in the chestnut blight fungus Cryphonectria parasitica. Canadian Journal of Botany. 69: 1407-1413.

Milgroom, M.G., Wang, K., Zhou, Y., Lipari, S.E. \& Kaneno, S. (1996). Intercontinental population structure of the chestnut blight fungus, Cryphonectria parasitica. Mycologia. 88: $179-190$

Milgroom, M.G., Sotirovski, K., Spica, D., Davis, J.E., Brewer M.T., Milev, M. \& Cortesi, P. (2008). Clonal population structure of the chestnut blight fungus in expanding ranges in southeastern Europe. Molecular Ecology. 17: 4446-4448. 
Milgroom, M.G. \& Cortesi, P. (1999). Analysis of population structure of the chestnut blight fungus based on vegetative incompatibility genotypes. Proceedings of the National Academy of Sciences. 96: 10518-10523.

Milgroom, M.G. \& Cortesi, P. (2004). Biological control of chestnut blight with hypovirulence: A critical analysis. Annual Reviews of Phytopathology 42: 311-338.

Montenegro, D., Aguín, O., Sainz, M.J., Hermida, M. \& Mansilla, J.P. (2008). Diversity of vegetative compatibility types, distribution of mating types and occurrence of hypovirulence of Cryphonectria parasitica in chestnut stands in NW Spain. Forest Ecology and Management. 256: 973980.

Newhouse, J.R. \& MacDonald, W.L. (1991). Ultrastructure of hyphal anastomoses between vegetatively compatible and incompatible virulent and hypovirulent strains of Cryphonectria parasitica. Canadian Journal of Botany. 69: 602-614

Nuss, D.L. (2005). Hypovirulence: Mycoviruses at the fungal-plant interface. Nature Reviews Microbiology. 3: 632642. O

Nuss, D.L., Hillman, B.I., Rigling, D. \& Suzuki, N. (2006). Index of Viruses - Hypoviridae. En: Büchen-Osmond C (Ed) ICTVdB - The Universal Virus Database, version 4. Columbia University, New York, USA.

Parsley, T.B., Chen, B., Geletka, L.M. \& Nuss, D.L. (2002). Differential modulation of cellular signaling pathways by mild and severe hypovirus strains. Eukaryotic Cell. 1: 401-413.

Paul, C.P. \& Fulbright, D.W. (1988). Double-stranded RNA molecules from Michigan hypovirulent isolates of Endothia parasitica vary in size and sequence homology. Phytophatology. 78: 751-755.

Peever, T.L., Liu, Y.C. \& Milgroom, M.G. (1997). Diversity of hypoviruses and other double-stranded RNAs in Cryphonectria parasitica in North America. Phytophatology. 87: 1026-1033.

Peever, T.L., Liu, Y.C., Wang, K., Hillman, B.I., Foglia, R. \& Milgroom, M.G. (1998). Incidence and diversity of doublestranded RNAs ocurring in the chestnut blight fungus Cryphonectria parasitica, in China and Japan. Phytophatology. 88: 811-817.

Perlerou, C. \& Diamandis, S. (2006). Identification and geographic distribution of vegetative compatibility types of Cryphonectria parasitica and ocurrence of hypovirulence in Greece. Forest Phytophatology. 36: 413-421.

Polashock, J.J. \& Hillmanl, B.I. (1994). A small mitochondrial double-stranded (ds) RNA element associated with a hypovirulent strain of the chestnut blight fungus and ancestrally related to yeast cytoplasmic $T$ and $W$ dsRNAs. Proceedings of the National Academy of Sciences. 91: 8680-8684.

Prospero, S., Conedera, M., Heiniger, U. \& Rigling, D. (1998). Sopravivenza e sporulazione di Cryphonectria parasitica su legna di Castanea sativa depositata in bosco. Edagricole. Monti e Boschi. 3/4: 44-50.
Radocz, L. (2001). Study of subpopulations of the chestnut blight (Cryphonectria parasitica) fungus in the Carpathian basin. Forest Snow and Landscape Research. 76: 368-372.

Rigling, D., Heiniger, U. \& Hohl, H.R. (1989). Reduction of laccase activity in dsRNA containing hypovirulent strains of Cryphonectria (Endothia) parasitica. Phytophatology. 79: 219-223.

Roane, M., Griffin, G. \& Elkins, J. (1986). Chestnut blight, other Endothia diseases, and the genus Endothia. APS Press, St. Paul, MN.

Robin, C. \& Heiniger, U. (2001). Chestnut blight in Europe: diversity of Cryphonectria parasitica, hypovirulence and biocontrol. Forest Snow and Landscape Research. 76: 361367.

Robin, C., Anziani, C. \& Cortesi, P. (2000). Relationship between biological control, incidence of hypovirulence and diversity of vegetative compatibility types of Cryphonectria parasitica in France. Phytophatology. 90: 730-737.

Robin, C., Capdevielle, X., Martin, M., Traver, C. \& Colinas, C. (2009). Cryphonectria parasitica vegetative compatibility type analysis of populations in south-western France and northern Spain. Plant Pathology. 58(3): 527-535.

Russin, J.S., Shain, L. \& Nordin, G.L. (1984). Insects as carriers of virulent and cytoplasmatic hypovirulent isolates of the chestnut blight fungus. Journal of Economic Entomology. 77: 838-846.

Saupe, S.J. (2000). Molecular genetics of heterokaryon incompatibility in filamentous ascomycetes. Microbiology and Molecular Biology Reviews. 64: 489-502.

Scheffer, R.P. (1997). The nature of disease in plants. Cambridge University Press, Cambridge.

Seemann, D., Bouffier, V., Kehr, R., Wulf, A., Schroder, T., Unger, J. (2001). Die Esskastanie (Castanea sativa Mill.) in Deutschland und ihre Gefährdung durch den Kastanienrindenkrebs (Cryphonectria parasitica [Murr.] Barr). Nachritenblatt des Deutschen Pflanzenschutzdienstes. 53: 49-60.

Shapira, R., Ghoil, G.H., Hillman, B.I. \& Nuss, D.L. (1991a). The contribution of defective RNAs to the complexity of viralencoded double-stranded RNA populations present in hypovirulent strains of the chestnut blight fungus Cryphonectria parasitica. EMBO Journal. 10 (4): 741-746.

Shapira, R., Ghoi, G.H. \& Nuss, D.L. (1991b). Virus-like genetic organization and expression strategy for a doublestranded RNA genetic element associated with biological control of chestnut blight. EMBO Journal. 10 (4): 731-739.

Sivanesan, A. \& Holliday, P. (1981). Cryphonectria parasitica. CMI Descriptions of Pathogenic Fungi and Bacteria, No 704. CAB International, Wallingford, Reino Unido.

Smart, C.D., Yuan, W., Foglia, R., Nuss, D.L., Fulbright, D.W. \& Hillman, B.I. (1999) Cryphonectria hypovirus 3, a virus species in the family Hypoviridae with a single open reading frame. Virology. 265: 66-73. 
Sotirovski, K., Milgroom, M.G., Rigling D. \& Heiniger, U. (2006). Occurrence of Cryphonectria hypovirus 1 in the chestnut blight in Macedonia. Forest Pathology. 36:136-143.

Sotirovski, K., Papazova-Anakieva, I., Grünwald, N.J. \& Milgroom, M.G. (2004). Low diversity of vegetative compatibility types and mating type of Cryphonectria parasitica in the Southern Balkans. Plant Pathology. 53: 325-333.

Trestic, T., Uscuplic, M., Colinas, C., Rolland, G., Giraud, A. \& Robin, C. (2001). Vegetative compatibility type diversity of Cryphonectria parasitica populations in BosniaHerzegovina, Spain and France. Forest Snow and Landscape Research. 76: 391-396.

Turchetti, T., Ferretti, F. \& Maresi, G. (2008). Natural spread of Cryphonectria parasitica and persistence of hypovirulence in three Italian coppiced chestnut stands. Forest Pathology. 38 (4): 227-243.

Van Alfen, N.K., Jaynes, R.A., Anagnostakis, S.L. \& Day, P.R. (1975). Chestnut blight: Biological control by transmissible hypovirulence in Endothia parasitica. Science. 189: $890-891$.
Valdezate, C., Alzugaray, R., Landeras, E. \& Braña, M. (2001). Situación actual de Cryphonectria parasitica (Murrill) Anderson, cancro cortical, en los castañares asturianos. Boletín de Sanidad Vegetal Plagas. 27: 401-410.

Wang, P. \& Nuss, D.L. (1995). Induction of a Cryphonectria parasitica cellobiohydrolase I gene is suppressed by hypovirus infection and regulated by a GTP-binding proteinlinked signaling pathway involved in fungal pathogenesis. Proceedings of the National Academy of Sciences USA. 92: 11529-11533.

Wang, K., Shao, J. \& Lu, J. (1991). On vegetative compatibility of Cryphonectria parasitica in Jiangsu and Anhui. Journal of Nanjing Agricultural University. 14: 44-48.

Wendt, R., Weidhaas, J., Griffin, G.J. \& Elkins J.R. (1983). Association of Endothia parasitica with mites isolated from cankers on American chestnut trees. Plant Disease 67: 757758.

Zhang, L., Baasari, R.A. \& Van Alfen, N.K. (1998). Viral repression of fungal pheromone precursor gene expression. Molecular and Cellular Biology. 18: 953-959. 